\title{
Psychosocial approaches to violence and aggression: contextually anchored and trauma-informed interventions
}

\author{
Deborah Horowitz, ${ }^{1}$ Margaret Guyer, ${ }^{2,3,4}$ and Kathy Sanders ${ }^{5,6 *}$
}

\author{
${ }^{1}$ Office of Training and Development, Massachusetts Department of Mental Health, Westborough, Massachusetts, USA \\ ${ }^{2}$ Central Office Research Review Committee, Massachusetts Department of Mental Health, Boston, Massachusetts, USA \\ ${ }^{3}$ Massachusetts Mental Health Center Division of Public Psychiatry, Boston, Massachusetts, USA \\ ${ }^{4}$ Harvard Medical School/Beth Israel Deaconess Medical Center, Boston, Massachusetts, USA \\ ${ }^{5}$ Clinical and Professional Services, Massachusetts Department of Mental Health, Boston, Massachusetts, USA \\ ${ }^{6}$ Harvard Medical School/Massachusetts General Hospital, Boston, Massachusetts, USA
}

Psychosocial interventions are part of the complex understanding and treatment of violent behavior in our state mental health hospitals. A comprehensive assessment of violence and aggression includes attention to all 3 domains of prevention and assessment (primary-institutional, secondary-structural, and tertiary-direct). Trauma experiences and their consequences may include behavioral violence and aggression. The authors' premise is that trauma is a universal component in the individual assessment of violent behavior. Therapeutic interventions must include a traumainformed formulation to be effective. Organizational commitment to trauma-informed, person-centered, recoveryoriented (TPR) care is crucial to the efficacy of any of the interventions discussed. Thus, the dynamic nature of the individual, interpersonal, environmental, and cultural factors associated with the daily operations of the inpatient unit need to be assessed through the lens of primary and secondary violence prevention, building on the recognition that the majority of persons served and staff have significant trauma histories. Once a compassionate, respectful, empathic, and empowering approach is embraced by leadership and staff, the work with individuals can proceed more effectively. Interventions used include a variety of cognitive-behavioral, interpersonal, and somatosensory therapies. These interventions, when effectively applied, result in more self-esteem, self-mastery, self-control for the person served, and diminished behavioral violence.

Received 29 January 2015; Accepted 9 March 2015

Key words: Aggression, prevention, psychosocial, trauma informed, violence, violence assessment.

\section{Introduction}

Across the United States, state mental health authorities are working to incorporate the voice of the person served into the mission and operating procedures of mental health services. The focus on providing traumainformed, person-centered, recovery-focused (TPR) care has gone hand in hand with initiatives to reduce restraint and seclusion episodes in psychiatric hospitals. State inpatient psychiatric facilities have a special opportunity to facilitate recovery in a trauma-informed, personcentered way and to foster positive growth and change

\footnotetext{
* Address for correspondence: Kathy Sanders, Massachusetts Department of Mental Health, 25 Staniford Street, Boston, MA 02114, USA.

(E-mail: kathy.sanders@massmail.state.ma.us)
}

in individuals who have traditionally been underserved and are in acute and/or enduring phases of their illnesses. These facilities, with access to diverse, flexible resources and modalities of care, are a vital part of any integrated continuum of care. ${ }^{1}$

Our mission as health care providers is to assist those seeking services to "turn pain into purpose."2 For those who express their pain through violence, our challenge is to help them transform that pain rather than meet it with the violence of restraint, seclusion, and coercion. Trauma engenders feelings of fear, anger, and shame, and violence is but one outward expression of those feelings. Violence, be it toward self or others, is multideterministic in nature, and as such requires interventions tailored to the individual and his or her own idiosyncratic behavioral expressions. Moreover, the episodes of violence in our 


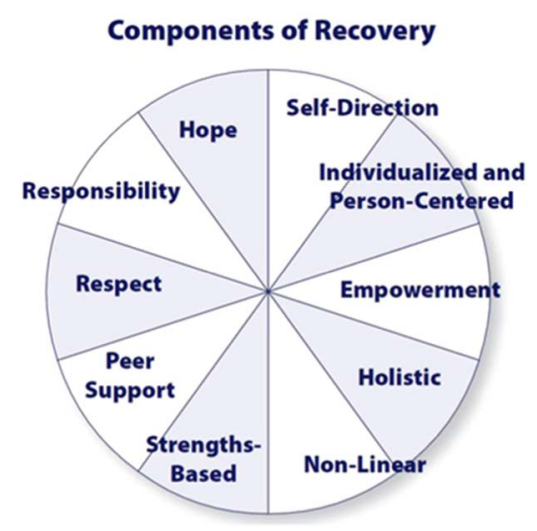

FIGURE 1. SAMHSA components and principles of recovery for individuals and organizations.

inpatient psychiatric units jeopardize the healing environment necessary to provide effective treatment and to empower the person served to be an active participant in his or her recovery. Staff and the person served suffer when violence occurs in what should be an environment of hope and opportunity for healing and change. As state mental health authorities across the country work to adopt TPR systems of care, implementation has often been met with anger and fear that these philosophical changes are at the expense of frontline staff members' ability to maintain safety for themselves and for those served. ${ }^{3}$ The high rates of injury in behavioral healthcare settings, particularly among frontline staff, undermine the quality of the milieu and efficacy of the therapeutic alliance so necessary to recovery.

This article seeks to explore the ways in which TPRinformed psychosocial interventions can ameliorate the negative impact of violence for persons served and for staff (see Figure 1). The authors will discuss how, given this lens, comprehensive assessment is conducted and appropriate treatment engagement, including accurate choice of psychosocial interventions, are facilitated. We will utilize a threat assessment approach that is organized around the identification, assessment, and de-escalation of threatening situations and is grounded in the TPR paradigm. The authors will pay particular attention to those psychosocial interventions that ultimately result, not only in the absence of violent behavior, but in safety, hope, and healing for all.

\section{Trauma-Informed Perspective}

There is growing recognition that trauma is widespread, and that the experience of trauma has far-reaching effects on a person's physical and mental well-being. The Adverse Childhood Experience (ACE) Study, utilizing a 10-item survey, found a powerful relationship between the number of traumatic incidents individuals

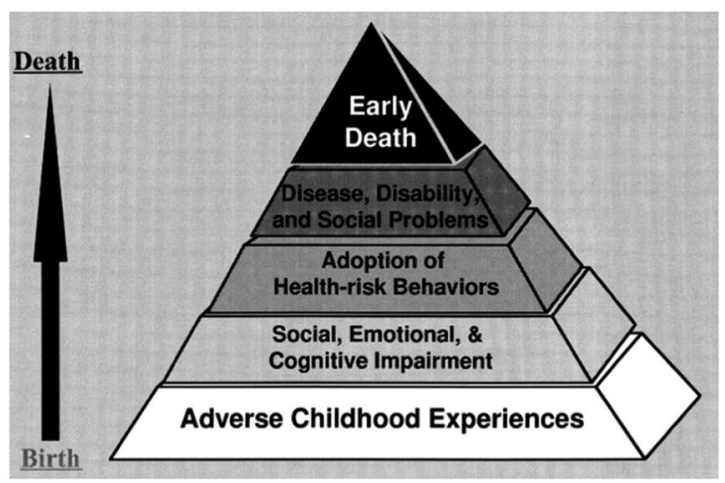

FIGURE 2. Conceptual understanding of a common pathway from adverse childhood experiences through impairment, risky behaviors, and disease to premature mortality. Public domain from Centers for Disease Control.

had experienced prior to the age of 18 and their developmental challenges and chronic health conditions. ${ }^{4}$ The items of the original ACE survey underscore that not only is trauma historical in nature, but it is also largely interpersonal, eg, physical or sexual abuse at the hands of a family member or disrupted attachments due to parental death, incarceration, or substance abuse.

The TPR approach to treating violence starts with the recognition that the behaviors that lead to inpatient psychiatric care, eg, violence to self or other, are a person's best attempt to communicate or mitigate the innumerable effects of the traumas they have experienced (see Figure 2). Among people with severe mental illness, the sheer pervasiveness of trauma, estimated to be as high as $90 \%$, is staggering and requires us to assume that trauma is a universal experience among people served on our state psychiatric inpatient units. ${ }^{5}$ The implication is that more than $90 \%$ of persons served have suffered some form of significant pain, loss, and fear, often at the violent hands of another. Assessing and treating violence as a direct consequence of trauma is but the first step on that journey of recovery.

\section{Assessment}

Equally central to TPR is the observation that "violence is a process, not an act." ${ }^{\prime 6}$ In order to accurately address violent behavior for a particular individual, a wider lens is needed to assess and understand the context and risk for violence. The public health model of violence defines 3 levels of violence: organizational/institutional (eg, stigma, discrimination); structural (eg, unemployment, involuntary hospitalization); and direct (eg, trauma $)^{7}$ (See Table 1). Violence is the synergistic culmination of individuals interacting with each other and the environment, each operating from their cultural affiliations and status within society overall, their relationships and trauma history, and their individual intrapsychic 
TABLE 1. Correlation of public health and threat aggression

prevention models

Public health prevention model Threat assessment prevention model

Primary Institutional Universal

Secondary Structural Identifying those at risk

Tertiary Direct Intervening an act of violence

schemas. ${ }^{8}$ Inpatient psychiatric units comprise miniature communities within which all 3 levels of violence (institutional, structural, and direct) are operating and relevant. A narrow focus on direct violence with little regard to the contributions of institutional and structural violence limits the efficacy of any intervention or treatment modality.

Within the field of violence risk assessment, the concept of threat assessment focuses on preventing violence within a community or institution. Threat assessment, consistent with the public health prevention model of violence, is organized into 3 levels of prevention: (1) primary (creating a community-wide climate that mitigates institutional violence and supports the commitment to nonviolence, (2) secondary (reducing structural violence by identifying and intervening with people at risk), and (3) tertiary (utilizing violencespecific interventions with people who are persistently violent).

\section{Primary prevention at the culture of care level}

The National Association of State Mental Health Program Directors (NASMHPD) has been the leader in emphasizing that the path to reducing violence on inpatient psychiatric units is to transform the authoritarian, controlling culture, which is too common among inpatient units, into a TPR culture. ${ }^{1,5}$ NASMHPD clearly places the locus of change within the organizational culture, as the mechanism necessary to reduce violence and associated restraint and seclusion episodes. ${ }^{5}$ A TPR culture of care may ameliorate the larger socioeconomic and cultural effects of institutional and structural violence, and thus reduce triggers for direct violence. Organizational expectations, values, and actions can influence the exhibition of challenging, aggressive, or violent behavior or they can encourage its prohibition. When a unit is motivated and committed to making changes, interventions are even more effective at reducing rates of violence. Fortunately, even when unit motivation is low, reduced violence rates are observed as a consequence of changes in the culture of care. ${ }^{3,9}$

1. Leadership commitment to TPR culture of care In creating a TPR care delivery environment, the very process of culture change must reflect these principles across all organizational levels.
Successful systems have engaged organizational leadership, frontline staff, and persons served in the process of direct change. ${ }^{3}$ Staff supported in the pursuit of their own growth and development activities can foster this in others. ${ }^{10}$ Also, those who are invested in the agency's mission are more likely to remain consistent with the interventions, modalities, and protocols associated with the overarching vision. ${ }^{5}$

2. Staff training

a. Given the pervasiveness of trauma, one of the first steps in the promotion of TPR culture of care is staff training. Staff should receive training on the etiology and universality of trauma, trauma's neurobiological sequelae, and implications for the nature and timing of early intervention and de-escalation strategies. ${ }^{11,12}$

b. Since the therapeutic alliance is integral to recovery, recovery-based communication styles must be taught. Staff who utilize therapeutic approaches of compassion, empathy, and respect reduce violence motivated by fear, anger, and shame. ${ }^{13}$ Training in low Expressed Emotion communication style, eg, avoiding verbal and nonverbal criticism, hostility, or over-intrusive, paternalistic attitudes; using supportive and nonthreatening methods for limit-setting; and emphasizing positive rather than negative communications, has been associated with a reduction in rates of restraint and seclusion. ${ }^{3}$

c. Staff should be trained to respond to an individual who is experiencing distress in order to avert escalation and possible violence. Utilization of noncoercive de-escalation and crisis intervention techniques is associated with reduced violence and fewer episodes of restraint or seclusion. ${ }^{14-17}$

d. Safety, Hope, and Healing (SHH) is a model developed by the Massachusetts Department of Mental Health for training staff that assumes that trauma is pervasive and influences many aspects of human behavior including violence. $\mathrm{SHH}$ involves 4 key components: (1) knowing the individual, (2) knowing the environment, (3) knowing yourself, and (4) knowing society. Importantly, $\mathrm{SHH}$ empowers staff to understand their own histories, triggers, and risk factors, as well as those of persons served, as a vehicle for identifying and mitigating potentially dangerous situations. These tools enable staff to better understand, make meaning of, and predict violence, thereby increasing personal and organizational safety and fostering hope for both staff and persons served. This approach supports an individualized formulation and treatment plan for recognizing and responding to the contextual fluctuations in risk for violence, and allows for an 
accurate contextually based evaluation of historical, intrapsychic, and interpersonal factors at play in any given interaction at any given moment.

3. Staff supervision and support

Staff members require ongoing training, supervision, and support in order to respond effectively to a person's level of distress. The intensity of people's need, despair, fear, shame, loneliness, or rage can be overwhelming, and may lead staff to recoil and distance themselves from their own intolerable feelings, which are often engendered by fear of professional inadequacy or fear for personal safety. Psychosocial interventions for staff include trauma-informed supervision, tertiary trauma and employee assistance services, and self-care and wellness activities that replenish staff and lead to higher work satisfaction. These interventions result in fewer negative encounters, including violence, as the result of staff compassion fatigue, vicarious trauma, and burnout. ${ }^{10}$

4. Modifying environmental factors

Violent incidents are lower on units where staff members have clear job descriptions and role expectations, and when staff are visibly present and actively engaged on the floor rather than sequestered in the nurses' station. The physical presence of unit and organizational leaders and predictable schedules filled with meaningful activities are associated with lower rates of violence. ${ }^{18}$

5. Systematic review of violent episodes

Systematic attention and review of past violent episodes have been associated with reductions in future violent episodes. Multidisciplinary consultation teams, with an eye toward reducing violence, consult on persons served who repeatedly exhibit challenging or unsafe behaviors, or who have had multiple episodes of seclusion or restraint. Formal debriefing of the involved persons served and staff is especially effective when a peer specialist participates in the debriefing. The use of multidisciplinary feedback teams who review each violent incident for alternatives to restraint and seclusion decreases unwarranted restraint and seclusion episodes. ${ }^{3}$

\section{Secondary prevention at the culture of care level}

In a TPR culture of care, secondary prevention efforts target the reduction of violence through identifying people at higher risk for violence, intervening to treat factors that increase vulnerability to violence, and monitoring risk status regularly and persistently over time. A comprehensive violence prevention assessment is ongoing and utilizes standardized measures; clinical observation; and collaboration, engagement, and empowerment with the person served. ${ }^{19,20}$ Staff monitor both the universal indicators for potential escalation, as well as an individual's specific contextual triggers and indicators of escalating risk. When done in collaboration with the person served, this model proves to be more effective as individuals learn to accurately assess their own ongoing fluctuations in stress, triggers, and coping resources.

1. Standardized assessment of universal risk factors A person is most at risk for violent behavior in the first 3 to 4 days of admission. ${ }^{9,21}$ A standardized violence risk assessment, eg, the Broset Violence Checklist, is most effective, given that the person served is new and not well known or understood by staff. $^{22}$ Systematic risk assessment upon admission can differentiate people at greatest risk for violent behavior. ${ }^{23}$ Systematic and frequent risk monitoring (eg, twice daily) during the first 3-4 days of a person's admission is associated with decreased rates of violent episodes. ${ }^{24,25}$

The formalization of risk assessment procedures affects the culture of a unit in several intangible ways as well. Regular review of the risk assessments promotes more effective intra- and inter-team communication and treatment planning. Staff knowledge and attention to the universal indicators of violence risk is increased and the direct focus on early intervention supports the use of more effective strategies for de-escalation. ${ }^{9}$ In one study, the number of severely aggressive incidents was unaffected; however, staff assessed these incidents as less severe, suggesting that systematic risk assessment increased staff's confidence in their ability to manage the risk for violence. ${ }^{16}$

2. Individualized formulations of violence risk Traditional, systematic risk assessment is integral to violence prevention efforts, but must be supplemented with other modalities and types of assessment in order to increase efficacy. With TPR systems of care, violent behavior is conceptualized as a "message," be it to the self or others. In order to understand the meaning of a person's violent behavior, a multidisciplinary approach is necessary to uncover the connections between the individual, the situation at hand, the setting, and the target of the violence. Attention to the relevant institutional and structural violence a person is subject to further informs this formulation. It requires a more dynamic focus and "an investigative, skeptical, inquisitive mindset."

3. Therapeutic alliance

A powerful vehicle for preventing adverse events like violence is knowing the individual based on a nonstigmatizing therapeutic relationship built 
TABLE 2. Common cognitive behavioral treatment components*

\begin{tabular}{|c|c|}
\hline Cognitive skills & $\begin{array}{l}\text { Training on general thinking and decision-making skills such as to stop and think before acting, generate alternative solutions, evaluate consequences, } \\
\text { and make decisions about appropriate behavior }\end{array}$ \\
\hline Cognitive restructuring & Activities and exercises aimed at recognizing and modifying cognitive distortions and errors that trigger violence \\
\hline $\begin{array}{l}\text { Interpersonal problem } \\
\quad \text { solving }\end{array}$ & Training in problem-solving skills for dealing with interpersonal conflict and peer pressure \\
\hline Social skills & Training in prosocial behaviors, interpreting social cues, taking other persons' feelings into account \\
\hline Anger control & Training in techniques for identifying triggers and cues that arouse anger and maintaining self-control \\
\hline Relapse prevention & Training on strategies to recognize and cope with high-risk situations and halt the relapse cycle before lapses turn into full relapses \\
\hline Moral reasoning** & Activities designed to improve the ability to reason about right and wrong behavior and raise the level of moral development \\
\hline Victim impact** & Activities aimed and getting people to consider the impact of their behavior on others \\
\hline
\end{tabular}

${ }^{*}$ Adapted from Lipsey et al $(2007)^{43}$.

** Violence-specific interventions

over time. Through this relationship, staff are aware of the meaningful aspects of an individual's history and are able to predict potential triggers, recognize early signs of difficulty, and utilize individual-specific interventions to avert escalation. Through this lens, the question becomes how to understand an individual's current presentation against the backdrop of "what happened to you?" rather than "what's wrong with you?" and shifts the paradigm even further to asking "what's strong in you?"26

4. Collaborative monitoring

A person's risk status is fluid; it is not a static state. At any given moment, an individual's level of risk is specific to his/her present emotional state, motivational attributions, interpersonal reactivity, internal coping mechanisms, external sources of support, and the level of distress and hopelessness he/she is experiencing. Staff and persons served can utilize knowledge of the individualized formulation of violence risk to collaborate in the monitoring and early intervention process.

\section{Psychosocial Interventions to Address Risk Factors Associated with Violence}

Psychosocial interventions that address the underlying risk factors associated with risk for violence and foster a strength-based understanding of trauma are an essential component of secondary prevention of violence.

Treatment delivery in a TPR system of care is based on 2 core principles ${ }^{27}$ :

1. Delivery of treatment reflects systemic awareness of the pervasiveness of trauma, the recognition of the impact of trauma on the individual, and an organizational responsiveness to that knowledge.

2. Treatment includes an integrated continuum of trauma-specific modalities to address the immediate cognitive, somatic, and emotional distress experienced by the person served and to foster long-term hope and recovery.

\section{Cognitive behavior therapy (CBT)}

CBT is the most widely studied modality of psychotherapy in the field of violence prevention, although other forms, eg, psychodynamic psychotherapy, are also effective. ${ }^{28}$ Most CBT approaches emphasize the ability to (a) recognize internal and external triggers; (b) observe and manage one's cognitive and emotional responses; and (c) resolve interpersonal conflict and negotiate getting one's needs met. CBT helps the person served to recognize the power of his/her own thoughts, particularly interpersonal/relational triggers, cognitive distortions, and the possibility of alternative interpretations (see Table 2). As cognitions shift, people's associated feelings of inadequacy, inferiority, and hopelessness also shift, and the possibility of recovery increases. CBT components that address the psychological factors that underlie violence, including fear, anger, and shame, empower an individual to resume a self-regulated life course.

\section{Sensory modulation interventions}

More recently, there has been increased recognition that in order for most CBT to work, one must include attention to the body because people who are physiologically aroused have difficulty utilizing cognitive strategies. ${ }^{29}$ People with mental illness are frequently less aware of their own physiological and sensory experiences. ${ }^{30}$ Identifying sensory triggers and physiological reactivity are essential to any effort to understand the antecedents of a violent episode and to develop person-specific strategies for early intervention and de-escalation. Teaching someone to recognize the changes in their own sensory experience enables them to more fully participate in the recovery process. Sensory modulation techniques allow for the creation of individualized tools to manage one's own physiological state. It is much easier for a person served to make their way in the world using personalized sensory 
tools under their conscious control than to rely on the presence of a staff member to do the same. Sensory modulation methods allow for choice and empowerment and decrease the sense of shame associated with being "less than" or "dependent." Empowering someone to use sensory strategies to self-regulate their level of arousal promotes self-efficacy. Inpatient units have introduced "sensory rooms" that provide a calming space with avenues for soothing and arousing each sense. ${ }^{31}$

\section{Interventions based on the presenting emotions}

\section{Shame}

Symptoms and behaviors that are triggered via a shamebased mechanism respond best to cognitive interventions within a "socio-ecological framework." 5 Skill-based modalities that target the need for control, mastery, and meaningful purpose foster pride and self-respect. Cognitive restructuring and enhancing therapies, motivational interviewing, stress management, vocational training, community outreach, and access to housing and higher education all counteract the negative messages that persons served have experienced via institutional and structural violence (see Table 3).

\section{Anger}

For those violent behaviors that are intra- and interpersonal in nature with a primary expression in the form of anger or rage, effective treatments include individual psychotherapy, dialectical behavioral therapy (with its focus on mindfulness, distress tolerance, and affect regulation), group therapies, co-occurring addiction work, anger management, and psycho-education regarding the interplay of trauma and mental illness. Intra- and interpersonal therapies foster the ability of the individual to self-reflect and to regulate the level of social interactions desired with a corresponding increase in positive social connections, where previous ones may have been damaged or non-existent.

Fear

Symptoms and violent behaviors that are primarily fearbased with a large global somatic component, including dissociation or hyper-arousal, respond best to interventions that target the brain stem and have a direct impact on sensation. Sensory modulation techniques, including sensory kits, comfort rooms, trauma-informed yoga, grounding and centering skills, progressive muscle relaxation, diaphragmatic breathing, eye movement desensitization and reprocessing (EMDR), and gentle reprocessing, to name a few, offer the fastest way to recalibrate the distorted fearful perceptions that lead to challenging, and even violent, behavior.

\section{Tertiary Prevention}

Treatments that address the underlying factors that give rise to violence are necessary but insufficient for the small minority of persons served (2-6\%) who engage in repeated episodes of violent behavior and are responsible for incidents of inpatient violence. ${ }^{32-34}$ The increasing demands for state mental institutions to serve people with forensic-related service needs in the absence of increased resources exacerbate concerns about the relevance of TPR in the treatment of violence. While acknowledging that approximately $41 \%$ of states' inpatient psychiatric funds are spent on people admitted with forensic related service needs, ${ }^{1}$ NASMHPD emphasizes that "all people served in state psychiatric hospitals [forensic or otherwise] should be considered in the process of recovery." ${ }^{11}$ Furthermore, while it is beyond the scope of this article to address the argument that persistent psychopathy in itself is a mental illness, there is burgeoning evidence that TPR is associated with violence reduction in forensic settings. Moreover, Skeem et $\mathrm{al}^{35}$ found that people with mental illness with high levels of psychopathy benefited as much from violence reduction treatments as people with mental illness without psychopathy.

\section{Psychosocial interventions to address violence}

Violence-specific interventions are necessary to decrease violence. ${ }^{36}$ While the known interventions that directly target aggression in adults appear to be nascent, the evidence-based research on children and adolescents

\section{TABLE 3. Relationship between trauma-induced feelings and appropriate psychosocial interventions}

\begin{tabular}{llll}
$\begin{array}{c}\text { Challenging } \\
\text { emotions }\end{array}$ & $\begin{array}{c}\text { Antidote } \\
\text { emotions }\end{array}$ & Interventions & Examples \\
\hline $\begin{array}{l}\text { Fear } \\
\text { Anger }\end{array}$ & $\begin{array}{l}\text { Compassion } \\
\text { Empathy }\end{array}$ & $\begin{array}{c}\text { Sensory modulation } \\
\text { Intra- and interpersonal } \\
\text { therapies } \\
\text { Skill mastery }\end{array}$ & $\begin{array}{c}\text { Grounding, sensory kits, body-based therapies, trauma-informed yoga, muscle relaxation, breath work, EMDR } \\
\text { DBT, individual therapy, group therapy, addiction work, anger management, mindfulness-based therapies }\end{array}$ \\
Shame & Pride & CBT, cognitive enhancing therapy, stress management, motivational interviewing, skills training, vocational \\
and educational training
\end{tabular}

EMDR = Eye Movement Desensitization and Reprocessing; DBT = dialectical behavior therapy; $C B T=$ cognitive behavioral therapy 
who are aggressive provides a general direction from which we can extrapolate to our adult, long-stay, inpatient population. Closely examining all risk factors for violence from the level of physiological arousal to historical, motivational, interpersonal, environmental, cultural, and societal indicators within a TPR context provides the best opportunity to decrease violence and foster hope and healing for the persons we serve. There are some trends in the research base that support TPR as the necessary context for effectively addressing violence, particularly adolescent interventions ${ }^{37,38}$ :

- Theoretical model: Treatments informed by a coherent theoretical model for understanding violence, its antecedents, and its consequences, and by clear principles that pervade the entire system of care are more likely to be effectively adopted. ${ }^{39}$

- Multimodal and multifocused: Treatments that utilize multiple modalities (eg, individual, group, family), target multiple domains (eg, emotion regulation, conflict resolution), and address larger system issues (eg, identity and roles within the family and the larger community) had significant effect sizes in contrast to the effect sizes of interventions with a singular focus, eg, a specific skill. ${ }^{40}$ CBT interventions that target anger control and interpersonal functioning show the most promise in reducing violent behavior. ${ }^{28,41-43}$

- Skilled clinical workforce: A well-trained, skilled clinical workforce providing clear, compassionate treatment is effective in reducing violence. ${ }^{39}$ The degree to which CBT is effectively implemented (eg, rigor of staff training and supervision in CBT) is positively related to effect size. ${ }^{43}$

The Mendota Juvenile Treatment Center (MJTC), located in a state psychiatric facility, is an example of a residential treatment for persistently violent adolescents that is comprehensive, multimodal, and system-based, and that shares many of the features that characterize TPR. The adolescents and their behavior are conceptualized in the context of their places within larger social systems (family, peer, community, etc.). Treatment focuses on the individual's alienation from "conventional life," which is understood as a product of both how others view and react to the individual and the individual's aggressive disdain and rejection of others. Treatment is designed to change the nature of interactions and to expand the roles and identities available to the adolescent. ${ }^{39}$ While the intervention includes skill training, the primary target is to increase the individual's openness and positive attitude to prosocial roles, identities, relationship dynamics, and life goals. ${ }^{44}$ When immediate family members are not able to participate, the treatment team works to engage extended family members or community members who may offer support. The MJTC approach resonates with the focus on recovery in the mental health system: the recognition that an expanded sense of self and self in relation to others is necessary for people with mental illness to cope with their illness and build meaningful lives. Five years postdischarge from MJTC, youth were $34 \%$ less likely to be rearrested and $50 \%$ less likely to commit a violent crime compared to those who did not participate in the MJTC program. $^{45}$

Aggressive replacement therapy (ART) is a CBT violence reduction program that originated as a treatment for adolescents and has also been found to be effective in working with adults and people with mental illness. ${ }^{46-49}$ ART is an intensive group treatment with multiple weekly sessions. Goldstein $e t a l^{46}$ conceptualize violence as comprising affective, behavioral, and values components, and the ART treatment modules of emotion regulation, social skills, and moral reasoning address each of these aspects of violence, respectively. The moral reasoning component asks participants to discuss moral and ethical questions and promotes mentalization skills, including the ability to recognize and imagine another person's experience. ART is associated with reductions in felony recidivism. ${ }^{43,50}$

Researchers at Children's Hospital in Boston have developed the RAGE-Control intervention, which recognizes the integral role of bodily focused interventions in the context of CBT and violence reduction. RAGE-Control is a video game that requires players to modulate their physiological arousal in order to play the game. Players who become physiologically hyperaroused effectively trigger the deactivation of their game control stick. The game settings are adjusted based on the individual's baseline arousal, and as the individual progresses, the settings can be set to present progressively more challenging goals of physiological modulation. Violence triggered by the need for power and control diminish as individuals master their own somatic processes and feel less at the mercy of their unregulated emotions. Preliminary data suggest that in conjunction with ACT, RAGE-Control may be associated with a decrease in violent episodes. ${ }^{51,52}$

\section{Putting It All Together}

Traumatologist and consultant Donna Riemer, RN, PPMHN-BC, reported on the efforts of a treatment team to reduce violence on a maximum security forensic mental health hospital unit using a trauma informed, sanctuary approach to (a) create cultural change in the milieu, (b) focus on consistent early interventions, and (c) develop working relationships between care providers and patients. ${ }^{53,54}$ The most secure maximum security unit in Wisconsin's state mental health system was the setting in which the treatment team leaders worked together with persons served to create a significant culture change to effectively embrace a zero-tolerance 
for violence. Persons served by this unit included those with major mental illness, personality disorders, and alcohol and other drug abuse, as well as medical and developmental disabilities, and most had significant legal issues. In order to do this, both staff and persons served had to understand their beliefs about and approach to reducing violence and barriers to change. The development of consistent rules, definitions, and approaches to violence was established collaboratively. With that consistency in place, better tools for early intervention and de-escalation of violence could be created in partnership with the person served. Emphasis on identifying and managing stressors that lead to violent behavior allowed for creative adoption of self-calming and modulating activities that served not only to de-escalate situations but to create a true healing sanctuary. Many of these activities have been highlighted in our discussion of specific interventions, including breath work, grounding techniques, meditation, progressive relaxation, and groups learning these techniques. Mixed psychoeducation groups of staff and persons served, regarding nonviolent communication as a means of expressing oneself appropriately and getting needs met, facilitated the desired culture change and consistency in approaching violent episodes. Additionally, groups that addressed anger management, cognitive and emotional regulation, coping and problem-solving, and conflict resolution, as well as other prosocial skills that respect the rights and boundaries of others as a basis for a trauma-informed environment of care, were embraced by all. ${ }^{53}$ Other changes incorporated included an emphasis on self-care and mutual support for the providers, as well as a robust debriefing process following all episodes of early intervention and de-escalation efforts.

The year-one results following the implementation of the culture change on this maximum security unit were striking. Compared to baseline episodes of violence from the year prior to the new approach, there was a $50 \%$ reduction in patient violence-related injuries, use of seclusion and restraint, emergency code calls, and complaints lodged by persons served. ${ }^{53}$ Moreover, lost days due to staff injuries were reduced by $62 \%$, and both staff and persons served reported an increase in feelings of safety. ${ }^{53}$

\section{Conclusion}

The medical model for understanding violence by parsing violent behaviors into psychotic, predatory, or impulsive (developmental/medical/injury) serves us well in determining medical interventions. ${ }^{34}$ There is a need to study and understand the history and experience of trauma in those with violent behaviors in order to provide evidencebased treatment and best practices to the treatment of individuals with aggressive and violent behavior. This will necessitate development of pilot programs that use trauma-informed approaches to violent behavior from primary, secondary, and tertiary prevention models. This comprehensive approach will serve both the individual and society at large. In that spirit, we call for primary prevention of violence in our society that supports community relationships, meaningful work, economic stability, and a trauma-informed perspective in all human service endeavors. More specifically, the organizational/ institutional commitment to a TPR orientation is crucial in the efficacy of any of the interventions discussed. In addition to the traditional means of assessing risk for violence, using the public health model of violence as a lens helps us to better understand and ameliorate symptoms that result from institutional, structural, and direct violence. The dynamic nature of the individual, interpersonal, environmental, and cultural factors associated with the daily operations of the unit need to be assessed through the lens of primary and secondary violence prevention, which recognizes that the majority of people served have significant trauma histories. Once a compassionate, respectful, empathic, and empowering approach is embraced by leadership and staff, the work with the individual can proceed more effectively. Interventions used include a variety of cognitive-behavioral, interpersonal, and somatosensory therapies. These interventions, when effectively applied, result in more selfesteem, self-mastery, and self-control, and diminished behavioral violence. This is the hope for the future efficacy of treatment in our state mental hospitals that combines a rigorous medical model with environmental and psychosocial interventions.

We recognize that fiscal constraints will limit the implementation of this approach, which requires a better trained and supervised work force. The value of time with individuals served in our inpatient institutions is part of the cost of this TPR approach, which is founded on interpersonal engagement and training of both staff and persons served. It is imperative that this change start at the top, and institutional leaders embrace this perspective. Since all institutions have training as part of an ongoing requirement for quality service provision, these efforts can be retooled to develop new ways to train staff and increase the skills required for implementing these psychosocial interventions. More pilot studies are needed that include standardized violence/aggression assessment and monitoring tools, while applying a TPR approach to reducing violence. These studies will create the evidence base needed for systematic culture change for behavioral health integration throughout the healthcare delivery system, our communities, and families to create a nontraumatizing society.

\section{Disclosures}

The authors do not have anything to disclose. 


\section{SUPPLEMENTARY MATERIAL}

To view supplementary material for this article, please visit http://dx.doi.org/10.1017/S1092852915000280.

\section{REFERENCES:}

1. Parks J, Radke AQ, Haupt MB. The Vital Role of State Psychiatric Hospitals. Alexandria, VA: National Association of State Mental Health Program Directors Medical Directors Council; 2014.

2. Colozzi E. Creating careers with confidence in EOHSS. Paper presented at the Aspiring Supervisor's Series, MA Executive Office of Health and Social Services, Westborough, MA, October 2014.

3. Espinosa L, Harris B, Frank J, et al. Milieu improvement in psychiatry using evidence-based practices: The long and winding road of culture change. Arch Psychiatr Nurs. In press. DOI: 10.1016/ j.apnu.2014.08.004

4. Anda RF, Felitti VJ, Walker J, et al. The enduring effects of abuse and related adverse experiences in childhood: a convergence of evidence from neurobiology and epidemiology. Eur Arch Psychiatry Clin Neurosci. 2006; 256(3): 174-186.

5. Substance Abuse and Mental Health Services Administration. Trauma-Informed Care in Behavioral Health Services. Treatment Improvement Protocol (TIP) Series 57. HHS Publication No. (SMA) 13-4801 Rockville, MD: Substance Abuse and Mental Health Services Administration; 2014.

6. Fein RA. Behavioral threat assessment and preventing targeted violence: history, opportunity, concerns. Paper presented at Violence Risk and Threat Assessment: An Historic and Dynamic Perspective, Boston, MA, November 2014.

7. Gilligan J. Violence: Our Deadly Epidemic and Its Causes. New York: GP Putman's Sons; 1996.

8. Tremblay RE. The development of aggressive behaviour during childhood: what have we learned in the past century? International Journal of Behavioral Development. 2000; 24(2): 129-141.

9. Abderhalden C, Needham I, Dassen T. Halfens, Halfens R, Haug HJ, Fischer JE. Structured risk assessment and violence in acute psychiatric wards: randomized controlled trial. The British Journal of Psychiatry. 2008; 193(1): 44-50.

10. Figley CR. Compassion Fatigue: Coping with Secondary Traumatic Stress Disorder in Those Who Treat the Traumatized. New York: Routledge; 1995.

11. Fisher D. A professional's lived experience. Trauma Informed Training at Massachusetts Department of Mental Health, Worcester Recovery Center and Hospital, Worcester, Massachusetts, August $20,2014$.

12. Uram S. The neurobiology of trauma made simple. Trauma Informed Training at MA Department of Mental Health, Worcester Recovery Center and Hospital, Worcester, Massachusetts, August 20, 2014.

13. Huckshorn KH. Reducing seclusion and restraint in mental health settings: core strategies for prevention. J Psychosoc Nurs Ment Health Serv. 2004; 42(9): 22-33.

14. Jambunathan J, Bellaire K. Evaluating staff use of crisis prevention intervention techniques: a pilot study. Issues Ment Health Nurs. 1996; 17(6): 541-558.

15. Richmond I, Trujillo D, Schmelzer J, Phillips S, Davis D. Least restrictive alternatives: do they really work? J Nurs Care Qual. 1996 11(1): 29-37.

16. Needham I, Abderhalden C, Meer R, et al. The effectiveness of two interventions in the management of patient violence in acute mental inpatient settings: Report on a pilot study. J Psychiatr Ment Health Nurs. 2004; 11(5): 595-601.

17. Richmond JS, Berlin JS, Fishkind AB, et al. Verbal de-escalation of the agitated patient: consensus statement of the American
Association for Emergency Psychiatry Project BETA De-escalation Workgroup. West J Emerg Med. 2012; 13(1): 17-25.

18. Katz P, Kirkland FR. Violence and social structure on mental hospital wards. Psychiatry. 1990; 53(3): 262-277.

19. McDermott BE, Holoyda BJ. Assessment of aggression in inpatient settings. CNS Spectr. 2014; 19(5): 425-431.

20. Monahan J, Skeem JL. The evolution of violence risk assessment. CNS Spectr. 2014; 19(5): 419-424.

21. McNeil DE, Binder RL, Greenfield TK. Predictors of violence in civilly committed acute psychiatric patient. Am J Psychiatry. 1988; 145(8): 965-970.

22. Abderhalden C, Needham I, Miserez B, et al. Predicting inpatient violence in acute psychiatric wards using the Brøset-ViolenceChecklist: a multicentre prospective cohort study. J Psychiatr Ment Health Nurs. 2004; 11(4): 422-427.

23. Newton VM, Elbogen EB, Brown CL, Snyder J, Barrick AL. Clinica decision-making about inpatient violence risk at admission to a public sector acute psychiatric hospital. J Am Acad Psychiatry Law. $2012 ;$ 40(2): 206-214.

24. McNiel DE, Binder RL. Predictive validity of judgments of dangerousness in emergency civil commitment. A J Psychiatry. 1987; 144(2): 197-200.

25. Grassi L, Peron L, Marangoni C, Zanchi P, Vanni A. Characteristics of violent behavior in acute psychiatric inpatients: a 5-year Italian study. Acta Psychiatr Scand. 2001; 104(4): 273-279.

26. Sharp C. On becoming trauma informed. The National Council for Behavioral Health. www.thenationalcouncil.org/events-andtraining/webinars/webinar-archive/. Relias Webinar, September 22,2014

27. Substance Abuse and Mental Health Services Administration. SAMHSA's Concept of Trauma and Guidance for a Trauma-Informed Approach. HHS Publication No. (SMA) 14-4884 Rockville, MD: Substance Abuse and Mental Health Services Administration; 2014.

28. Hockenhull JC, Whittington R, Leitner M, et al. A systematic review of prevention and intervention strategies for populations at high risk of engaging in violent behaviour: Update 2002-8. Health Technol Assess. 2012; 16(3): 1-152.

29. Linehan M. The Cognitive Behavioral Treatment of Borderline Personality Disorder. New York: Guilford Press; 1993.

30. Champagne T, Stromberg N. Sensory approaches in inpatient psychiatric settings: innovative alternatives to seclusion and restraint. J Psychosoc Nurs Ment Health Serv. 2004; 42(9): 35-43.

31. LeBel J, Stromberg N, Duckworth K, et al. Child and adolescent inpatient restraint reduction: a state initiative to promote strength based care. J Am Acad Child Adolesc Psychiatry. 2004; 43(1): 37-45.

32. Rasmussen $\mathrm{K}$, Levander S. Individual rather than situation characteristics predict violence in a maximum security hospital. J Interpers Violence. 1996; 11(3): 376-390.

33. Polaschek DLL, Collie RM. Rehabilitating serious adult violent offenders: an empirical and theoretical stocktake. Psychology, Crime and Law. 2004; 10(3): 321-334.

34. Stahl S. Deconstructing violence as a medical syndrome: mapping psychotic, impulsive, and predatory subtypes to malfunctioning brain circuits. CNS Spectr. 2014; 19(5): 357-365.

35. Skeem JL, Monahan JU, Mulvey EP. Psychopathy, treatment involvement, and subsequent violence among civil psychiatric patients. Law Hum Behav. 2002; 26(6): 577-603.

36. McGuire J. A review of effective interventions for reducing aggression and violence. Philos Trans R Soc Lond B Biol Sci. 2008; 363(1503): 2577-2597.

37. Tate DC, Reppucci ND, Mulvey EP. Violent juvenile delinquents: treatment effectiveness and implications for future action. Am Psychol. 1995; 50(9): 777-781.

38. Eyberg S, Nelson M, Boggs S. Evidence-based psychosocial treatments for children and adolescents with disruptive behavior. J Clin Child Adolesc Psychol. 2008; 37(1): 215-237. 
39. Caldwell MF, VanRybroek G. Effective treatment programs for violent adolescents: programmatic challenges and promising features. Aggression and Violent Behavior. 2013; 18(5): 571-578.

40. Garrido V, Morales LA. Serious (violent and chronic) juvenile offenders: A systematic review of treatment effectiveness in secure corrections. Campbell Systematic Reviews. 2007; 7. DOI: 10.4073/csr.2007.7.

41. Renwick SJ, Black L, Ramm M, Novaco RW. Anger treatment with forensic hospital patients. Legal and Criminological Psychology. 1997; 2(1): 103-116.

42. Frey REC, Weller J. Behavioural management of aggression through teaching interpersonal skills. Psychiatr Serv. 2000; 52(5): 607-609.

43. Lipsey MW, Landeberger NA, Wilson SJ. Effects of cognitive behavioral programs for criminal offenders. Campbell Systematic Reviews. 2007; 6. DOI: 10.4073/csr.2007.6.

44. Kornblum R. Violence Prevention Through Movement and Pro-Social Skills. Oklahoma City, OK: Wood and Barnes Publishing; 2002.

45. Caldwell MF. Treatment of adolescents with psychopathic features. In Kiehl KA, Sinnott-Armstrong WP, eds. Handbook on Psychopathy and Law. New York: Oxford University Press; 2013: 201-230.

46. Goldstein AP, Glick B, Rainer S. Aggression Replacement Training. Champaign, IL: Research Press; 1987.

47. Glick B, Gibbs JC. Aggression Replacement Training@: $A$ Comprehensive Intervention for Aggressive Youth, 3rd ed. Champaign, IL: Research Press; 2011.
48. McGuireJ, Clark D. A national dissemination program. In Goldstein AP, Nensen R, Daleflod RB, Kalt UM, eds. New Perspective on Aggression Replacement Training. Chichester, UK: Wiley; 2004: 139-150.

49. Hatcher RM, Palmer EJ, McGuire J, Hounsome JC, Bilby CAL, Hollin CR. Aggression replacement training with adult male offenders within community settings: a reconviction analysis. Journal of Forensic Psychiatry and Psychology. 2008; 19(4): 517-532.

50. Barnoski R. Washington State's Implementation of Aggression Replacement Training for Juvenile Offenders: Preliminary Findings. Olympia, WA: The Evergreen State College, Washington State Institute for Public Policy; 2002.

51. Kahn J, Ducharme P, Travers B, Gonzalez-Heydrich J. Rage control: Regulate And Gain Emotional control. Stud Health Technol Inform. 2009; 149: 335-343.

52. Ducharme P, Wharff E, Kahn J, Hutchinson Logan G. Augmenting anger control therapy with a videogame requiring emotional control: a pilot study on an inpatient psychiatric unit. Adolescent Psychiatry. 2012; 2(4): 323-332.

53. Riemer D. Creating sanctuary: reducing violence in a maximum security forensic psychiatric hospital unit. Forensic Nurses. 2009; 15(1): 302 .

54. Bloom S. Creating Sanctuary: Toward the Evolution of Sane Societies. New York: Routledge; 1997. 